\title{
Measurement of Level Density Parameters of Fission Fragments Following Thermal Neutron Induced Fission of Uranium-235
}

\author{
Katsuhisa NISHIO ${ }^{\dagger}$, Itsuro KIMURA, \\ Department of Nuclear Engineering, Kyoto University* \\ Yoshihiro NAKAGOME \\ Research Reactor Institute, Kyoto University**
}

(Received December 2, 1996)

\begin{abstract}
Level density parameters of fission fragments following the thermal neutron induced fission of ${ }^{235} \mathrm{U}$ were determined as a function of the fragment mass by measuring the energy and multiplicity of prompt neutrons from a specified mass. This measurement gave the absolute values of the parameters for the neutron-rich nuclei covering the mass range from 80 to $155 \mathrm{u}$ except the region from 115 to $125 \mathrm{u}$. The obtained parameters showed a saw-tooth trend against the mass with the minimum value around the double magic number, $132 \mathrm{u}$, and this trend was close to that for ${ }^{252} \mathrm{Cf}(\mathrm{s} . \mathrm{f})$ by Budtz-Jørgensen et al. The analysis by a phenomenological description of the level density parameters including the shell and collective effects suggested the existence of a collective motion of the fission fragment at the moment of prompt neutron emission.
\end{abstract}

KEYWORDS: neutron induced fission, uranium 235, fission fragments, prompt neutrons, level density parameters, collective motions, neutron multiplicity

\section{INTRODUCTION}

The nuclear level density (LD) is a fundamental quantity to describe the deexcitation of a highly excited nucleus by a statistical approach. The LD can be obtained ${ }^{(1)}$ from the measurement and its analysis of (1) the resonance capture of neutrons or charged particles, (2) the inelastic scattering of protons, neutrons, alpha particles or others, and (3) the Ericson fluctuation in compound nuclear reactions. Such measurement and analysis provide the LD at the corresponding excitation energy, and the gross dependence of the LD on the excitation energy is described by the level density parameter (LDP).

Based on the experimental data obtained from the above-mentioned measurement, the LDPs of a large number of nuclei have been derived ${ }^{(2)-(7)}$. In these works, an emphasis is put on the systematic variation of the LDPs against the mass number, $A$. The LDP increases linearly against $A$ as predicted by the Fermi-gas model. But, a few dips are observed along the line, and it is found that their positions apparently correspond to the nuclear magic numbers. It brings an idea that the LDP is very much influenced by the shell effect and that the

\footnotetext{
* Yoshida, Sakyo-ku, Kyoto 606-01.

${ }^{* *}$ Kumatori-cho, Sennan-gun, Osaka 590-04.

Corresponding author, Tel. +81-724-51-2375,

Fax. +81-724-51-2603, E-mail: knishi@rri.kyoto-u.ac.jp
}

dependence of LDPs on $A$ may be expressed by using the shell-correction energy of the nucleus, $\delta W$. With this idea, phenomenological expressions of the parameter have been proposed $^{(5)}$. In spite of the success to predict the trend of the LDP vs. $A$ by using $\delta W$, the absolute value strongly depends on the model adopted. For example, if we take the enhancement of the LD by the collective motion into account, which originates from the rotation and vibration of nucleus, the absolute value of the LDP is about $30 \%$ smaller than that without the collective enhancement ${ }^{(7)}$.

On the other hand, Budtz-Jørgensen et al. ${ }^{(8)}$ directly derived the LDPs of the fission fragments for the spontaneous fission of ${ }^{252} \mathrm{Cf}$ from the correlation measurement of the fission fragments and the prompt neutrons. In the measurement, they obtained the energy spectrum and multiplicity of the neutrons from the specified fragment mass $\left(m^{*}\right)$. Since the former and the latter are good indicators of the nuclear temperature $(T)$ and the excitation energy $\left(E_{e x}\right)$ of the fragment, respectively, the LDP of the specified fragment, $a$, can be determined from the relation, $E_{e x}=a T^{2}$. Their $a\left(\mathrm{~m}^{*}\right)$ curve shows a saw-tooth trend with the minimum value at $130 \mathrm{u}$, which is considered to reflect the shell effect of the fission fragments, since this mass number is very close to the double magic number of 132. This method to determine the LDP has the advantages: (1) giving the absolute value, (2) obtaining the data for the neutron-rich nuclei, and (3) cov- 
ering the data in a wide-mass range in one measurement. Their data are sometimes used for the input parameter values in the calculation of the statistical decay of excited fission fragments through particle and gamma-ray emission $^{(9)}$.

In the present paper, we have obtained the absolute LDP values of the fission fragments for ${ }^{235} \mathrm{U}\left(n_{t h}, f\right)$ by the same principle of Budtz-Jørgensen et al. The purpose of this work is to investigate whether the $a\left(m^{*}\right)$ for ${ }^{235} \mathrm{U}\left(n_{t h}, f\right)$ has the similar saw-tooth trend as observed for ${ }^{252} \mathrm{Cf}$ (s.f). Another purpose is to compare the measured LDP values with the calculations by the phenomenological model assuming the shell and collective effects by Iljinov et al. ${ }^{(7)}$ From the comparison the existence of the collective motion of the fission fragments is investigated.

\section{EXPERIMENT}

\section{Experimental Procedure}

The fragment-neutron correlation measurement for ${ }^{235} \mathrm{U}\left(n_{t h}, f\right)$ was carried out by using the slow neutrons from the Kyoto University Reactor. The experimental setup at the exit of the super mirror neutron guide tube is shown in Fig. 1. The details of the setup and the detectors are described elsewhere $\mathrm{e}^{(10)(11)}$.

In a vacuum fission chamber made of aluminum, an uranium target and two fission fragment detectors were mounted. The uranium target with $99.9 \%$ enriched ${ }^{235} \mathrm{U}$ was made by the electrodeposition of $\mathrm{UO}_{2}\left(\mathrm{NO}_{3}\right)_{2}$ on a nickel foil of $90 \mu \mathrm{g} / \mathrm{cm}^{2}$ thick, and the thickness was
$140 \mu \mathrm{g}{ }^{235} \mathrm{U} / \mathrm{cm}^{2}$.

The kinetic energy of the fission fragment 1 (FFl) was determined with a silicon surface barrier detector (SSBD) of $900 \mathrm{~mm}^{2}$ in active area. Another fragment (FF2) was detected with a position-sensitive parallel plate avalanche counter (PPAC) with an active diameter of $22 \mathrm{~cm}$, and its energy was obtained from the time difference between the signals from the SSBD and PPAC. The fragment flight direction, $\theta$, against the axis passing through the centers of both fragment detectors was determined by the incident position of the FF2 on the PPAC. Each fragment mass was derived from the momentum and mass conservation. The details of the calibrations and the corrections in the data analysis are seen in the references ${ }^{(10)(11)}$.

The prompt neutrons emitted from the FF1 were detected with a liquid organic scintillator (NE213) of $12.8 \mathrm{~cm}$ in diameter and $5.1 \mathrm{~cm}$ in thickness. The neutron energy was determined by the time-of-flight (TOF) method with the flight path length of $75.8 \mathrm{~cm}$, for which the start signal was triggered by the pulse from the SSBD induced by the FF1. To discriminate the prompt neutrons from the background gamma rays, the pulse shape discrimination technique was applied. The neutron emission angle from the FF1 was equal to the fragment flight direction $(\theta)$ in this setup. The neutron energy was transformed to that in the center-of-mass (c.m.) system of the coincided fragment by the kinematics using the fragment velocity and $\theta$. The neutron multiplicity for the specified fragment mass was obtained

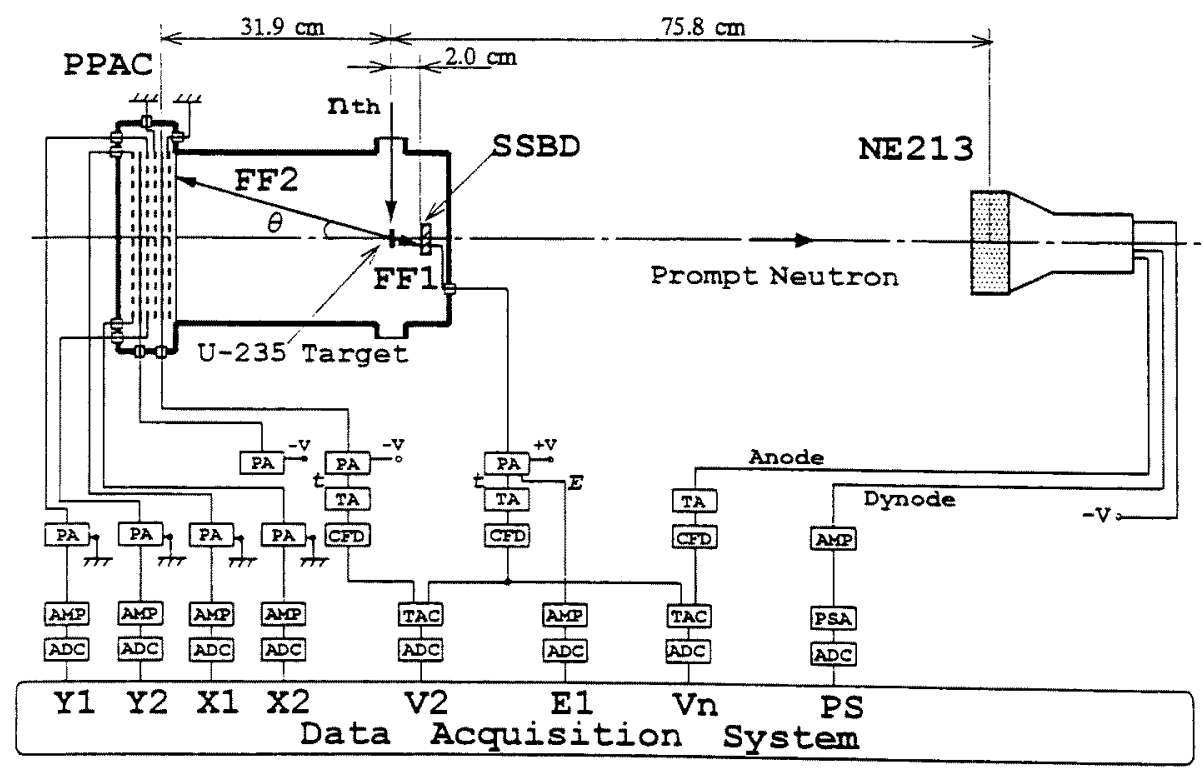

PA: Preamplifier

AMP: Amplifier

CFD: Constant-Fraction Discriminator PSA: Pulse Shape Analyzer

TAC: Time-to-Amplitude Converter ADC: Analog-to-Digital Converter

TA: Timing Amplifier

Fig. 1 Experimental setup of the fragment-neutron correlation measurement for ${ }^{235} \mathrm{U}\left(n_{t h}, f\right)$

Electronic circuits are also shown below, where the coincidence circuits are not depicted for simplicity. 
from the number of the fragment-neutron coincidences divided by the coincidence number of both fragments with a normalization of 2.43 per fission ${ }^{(12)}$. In order to obtain the neutron energy spectrum in the c.m. system and the multiplicity, the neutron detection efficiency influenced by the fragment motion was calculated by a Monte Carlo method.

\section{Experimental Results}

The obtained average neutron multiplicity as a function of the fragment mass, $\langle\nu\rangle\left(m^{*}\right)$, is shown in Fig. 2. For comparison, the data by Apalin et al. ${ }^{(13)}$, Maslin et al. ${ }^{\left({ }^{14)}\right.}$ and Boldeman et al. ${ }^{(15)}$ are depicted in the same figure. The present values are close to those by Maslin et al.

Typical neutron energy spectra in the c.m. system from the specified mass, $\phi(\eta)$, are shown in Fig. 3 in the form of $\phi(\eta) / \eta^{1 / 2}$ for selected eight mass bins. It is seen from this figure that the c.m. spectrum is represented by the following Maxwellian form ${ }^{(16)}$

$$
\phi(\eta)=\text { const. } \eta^{1 / 2} \exp \left(-\eta / T_{\text {eff }}\right),
$$

where $T_{\text {eff }}$ is the effective nuclear temperature and is related with the nuclear temperature as $T_{\text {eff }}=(8 / 9) T$. The fitted result with this equation is shown by the solid line.

The average energy of neutrons emitted from the specified mass, $\langle\eta\rangle\left(m^{*}\right)$, is shown in Fig. 4. It is worth noting that the shape of $\langle\eta\rangle\left(m^{*}\right)$ is of nearly bell-type and symmetric to the half mass division, whereas the $\langle\nu\rangle\left(m^{*}\right)$ distribution shows the saw-tooth shape.

\section{Level Density Parameter from NEUTRON MEASUREMENT}

The LDP relates the excitation energy and the temperature of the fragment by

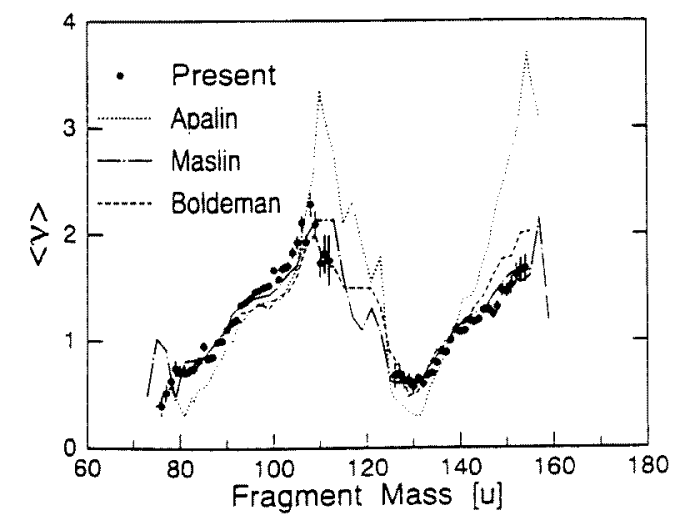

Fig. 2 Average neutron multiplicity as a function of fragment mass, $\langle\nu\rangle\left(\mathrm{m}^{*}\right)$

Present values (solid circles with statistical error bars) are compared with the data by Apalin et al. ${ }^{(13)}$ (dotted curve), Maslin et al. ${ }^{(14)}$ (dash-dotted curve) and Boldeman et al. ${ }^{(15)}$ (dashed curve).

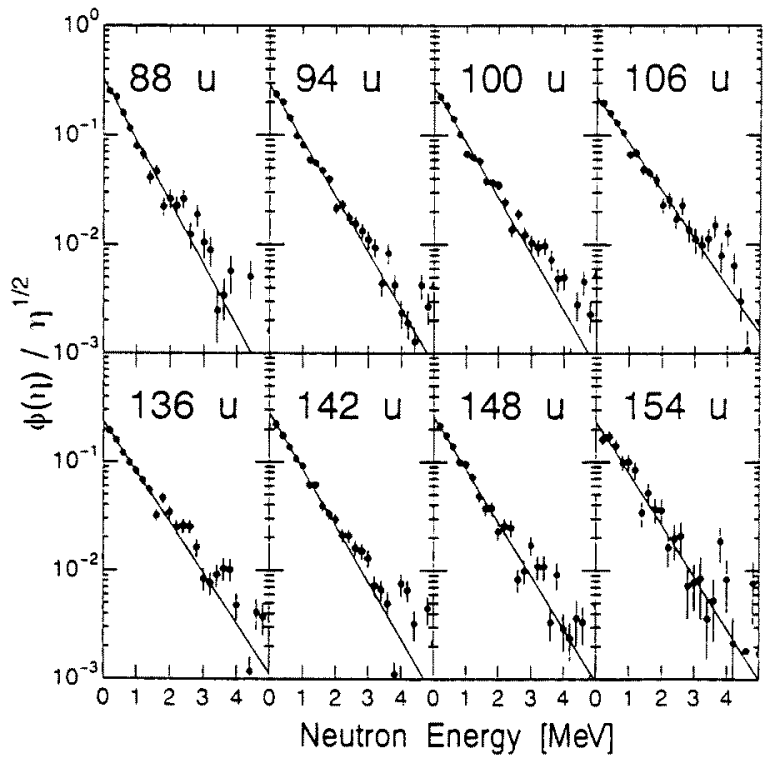

Fig. 3 Neutron energy spectra in the c.m. system for the selected mass bins are plotted in the form of $\phi(\eta) / \eta^{1 / 2}$.

Each spectrum is fitted to the Maxwellian spectrum described in the text (solid line).

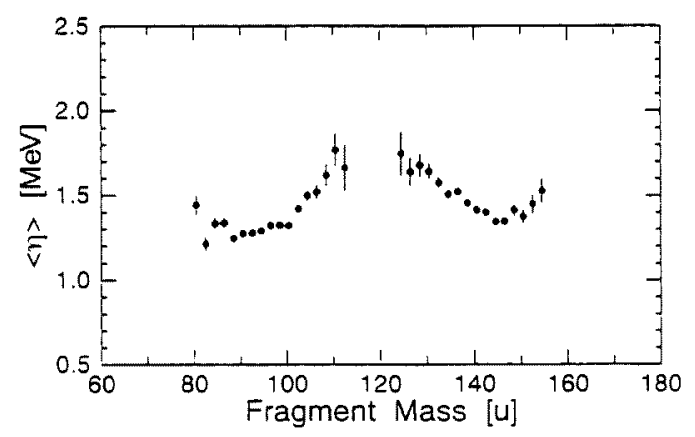

Fig. 4 Average neutron emission energy in the c.m. system as a function of fragment mass, $\langle\eta\rangle\left(m^{*}\right)$

$$
E_{e x}=a T^{2} \text {. }
$$

As described in the preceding section, the neutron spectrum in the c.m. system can be represented by the Maxwellian shape as Eq.(1). Therefore, the nuclear temperature of the fragment is estimated by using the average neutron emission energy as follows ${ }^{(16)}$ :

$$
T=(3 / 4)\langle\eta\rangle \text {. }
$$

The excitation energy of the fragment is determined by

$$
E_{\text {ex }}=\langle\nu\rangle\left\{\langle\eta\rangle+\left\langle B_{n}\right\rangle\right\}+E_{\gamma} .
$$

The first and second terms are the energies dissipated by the prompt neutrons and the gamma rays, respectively, and the latter is estimated ${ }^{(11)}$ by the half of the neutron binding energy, $\left\langle B_{n}\right\rangle$, as,

$$
E_{\gamma}=\left\langle B_{n}\right\rangle / 2 \text {. }
$$

We calculated $\left\langle B_{n}\right\rangle$ by using the mass table by Möller 
et $a .^{(17)}$, for which the most probable proton and neutron numbers were assumed. The excitation energy of the fragment vs. mass is shown in Fig. 5(a), and it varies between 7 and $20 \mathrm{MeV}$.

The obtained $a\left(m^{*}\right)$ for ${ }^{235} \mathrm{U}\left(n_{t h}, f\right)$ from Eqs.(2)-(5) is shown in Fig. 5(b). The errors in the figure contain (1) the statistical error and (2) the uncertainty due to the difference of the spectrum shape from the Maxwellian type. The latter means that if the shape of the neutron spectrum does not obey the Maxwellian type, the value of $T /\langle\eta\rangle$ is different from $3 / 4$, which brings the error in $T$ when the Eq.(3) is applied. The data in the Ref.(8) show that the deviation of this ratio is limited within about $10 \%$ of this value for most of the mass region, and hence this percentage is used to estimate the error. It is found that the $a\left(m^{*}\right)$ for ${ }^{235} \mathrm{U}\left(n_{t h}, f\right)$ shows a saw-tooth trend with the minimum value around $130 \mathrm{u}$, which reflects the strong shell effect. Regarding that the nuclear temperature varies only about $40 \%$ against the fragment mass (from Eq. (3) and Fig. 4), the saw-tooth shape of $a\left(m^{*}\right)$ is mainly brought from the distribution of the fragment excitation energy as shown in the upper part (a) of this figure. In other words, it can be said that the shape difference between the $\langle\nu\rangle\left(m^{*}\right)$ and the $\langle\eta\rangle\left(m^{*}\right)$ is the result of the saw-tooth trend of the LDP. In the same figure, $a\left(m^{*}\right)$ for ${ }^{252} \mathrm{Cf}$ (s.f) from Ref.(8) is also depicted. The shape of $a\left(m^{*}\right)$ for ${ }^{235} \mathrm{U}\left(n_{t h}, f\right)$ is close to that for ${ }^{252} \mathrm{Cf}(\mathrm{s}$.f). In particular, the minimum around $130 \mathrm{u}$ is commonly observed for
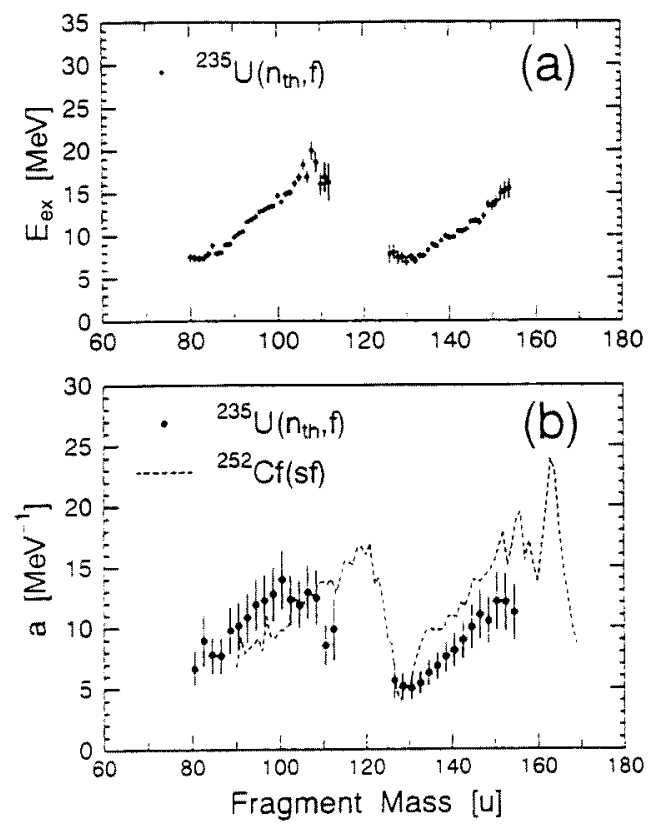

Fig. 5 (a) Excitation energies of fission fragments, $E_{e x}\left(m^{*}\right)$. (b) Level density parameter (LDP) of fission fragment for ${ }^{235} \mathrm{U}\left(n_{t h}, f\right)$ is plotted by solid circles with error bars.

The dotted curve is the LDP for ${ }^{252} \mathrm{Cf}(\mathrm{s.f})$ taken from Ref.(8). both fission systems. The values of $a\left(m^{*}\right)$ in the light fragment group for ${ }^{235} \mathrm{U}\left(n_{t h}, f\right)$ are slightly larger than that for ${ }^{252} \mathrm{Cf}$ (s.f), while in the heavy fragment group the data for ${ }^{235} \mathrm{U}\left(n_{t h}, f\right)$ lie below those for ${ }^{252} \mathrm{Cf}(\mathrm{s.f})$.

\section{Analysis and Discussion}

As discussed in the preceding section, the shell effect of the fragments influences the values of the LDP. For a phenomenological description of the LD, Iljinov et al. proposed an expression including the shell effect ${ }^{(7)}$. They analyzed the LDs of nuclei from neutron resonance results and others, and obtained the expression of LDP. In this process, they derived two sets of parameters $(\alpha, \beta, \gamma)$ in their LDP expression (following Eqs.(9)-(11)) on the basis of two assumptions, i.e., with and without consideration of the effect of collective motion.

The LD of a nucleus $\rho\left(E_{e x}\right)$ is described by ${ }^{(7)(18)}$

$$
\rho\left(E_{e x}\right)=K_{\text {rot }} K_{v i b} \rho_{\text {int }}\left(E_{e x}\right),
$$

where $K_{r o t}$ and $K_{v i b}$ are the coefficients for rotational and vibrational enhancements of the non-collective internal nuclear excitation $\rho_{\text {int }}\left(E_{e x}\right)$, respectively. The function $\rho_{\text {int }}\left(E_{e x}\right)$ is described by the following Fermigas expression:

$$
\begin{aligned}
\rho_{\text {int }}\left(E_{\text {ex }}\right)= & (1 / 12) \pi^{1 / 2} a^{-1 / 4} \\
& \cdot\left(E_{\text {ex }}-\Delta\right)^{-5 / 4} \exp \left[2\left\{a\left(E_{\text {ex }}-\Delta\right)\right\}^{1 / 2}\right] .
\end{aligned}
$$

The pairing energy $\Delta$ in $\mathrm{MeV}$ is described by ${ }^{(7)}$

$$
\Delta=12 \chi / A^{1 / 2},
$$

where $A$ is the mass number, and $\chi=0,1$ or 2 represents odd-odd, odd-even or even-even nuclei, respectively. The LDP is semiempirically expressed as follows by using the shell correction energy of a nucleus, $\delta W(Z, N)$, having $Z$ protons and $N$ neutrons.

$$
\begin{aligned}
& a\left(E_{e x}, Z, N\right) \\
& =\tilde{a}(A)\left[1+\delta W(Z, N) f\left(E_{e x}-\Delta\right) /\left(E_{e x}-\Delta\right)\right],
\end{aligned}
$$

where $f\left(E_{e x}\right)$ is a function giving the energy dependence of the LDP and becomes

$$
f\left(E_{e x}\right)=1-\exp \left(-\gamma E_{e x}\right),
$$

when $E_{e x}$ increases to infinity, the LDP converges to the following asymptotic value:

$$
\tilde{a}(A)=\alpha A+\beta A^{2 / 3} .
$$

The analytical expression of the rotational enhancement of the level density, $K_{r o t}$, and that of the vibrational enhancement, $K_{v i b}$, are described in Ref.(7). It should be noted here that $K_{\text {rot }}$ is unity when the nucleus is spherical, but for the deformed nucleus it has a value from 10 to 100 , and this value is much larger than the vibrational enhancement, $K_{v i b} \approx 3$.

Iljinov et al. obtained the three parameters $(\alpha, \beta, \gamma)$ in the above equations as $(0.090,-0.040,0.070)$ and $(0.114,0.098,0.051)$ when the collective effects were in- 
cluded $\left(K_{\text {rot }} \neq 1, K_{v i b} \neq 1\right)$ and not $\left(K_{\text {rot }}=K_{v i b}=1\right)$, respectively. It must be noted that these parameters were obtained with the shell-correction energy, $\delta W(Z, N)$, by Myers and Swiatecki (MS) ${ }^{(19)}$.

To adopt Eqs. (8)-(11) for the calculation of the LDPs of fission fragments, the following assumptions were made. First, the shell effect term in Eq.(9) was calculated by using the $\delta W\left(m^{*}\right)$ of MS in Fig. 6(a) and the $E_{e x}\left(m^{*}\right)$ in Fig. 5(a). For $\delta W$ of the fission fragments, the most probable proton and neutron numbers were assumed. Next, the value of $\chi$ in Eq.(8) is set to be 1 to evaluate the fragment paring energy $(\Delta)$, which is derived from the assumption that three types of nuclei (i.e. $\chi=0,1$ and 2 ) are included in the $m^{*}$-bin, but the $\Delta$ value averaged in this bin is equal to that of the oddeven nucleus. The calculated $a\left(m^{*}\right)$ for ${ }^{235} \mathrm{U}\left(n_{t h}, f\right)$ are shown in Fig. 6(b). In this figure, two kinds of plots depicted by the lozenges and squares are the results with and without the collective effects, respectively. It is clear from this figure that the $a\left(m^{*}\right)$ with the collective effects is smaller than that in the non-collective case, which means that the internal level density $\rho_{\text {int }}\left(E_{e x}\right)$ is reduced by introducing the collective enhancement. Also shown in Fig. 6(b) by dash-dotted and solid lines are the asymptotes of $a\left(m^{*}\right)$ calculated from Eq.(11).
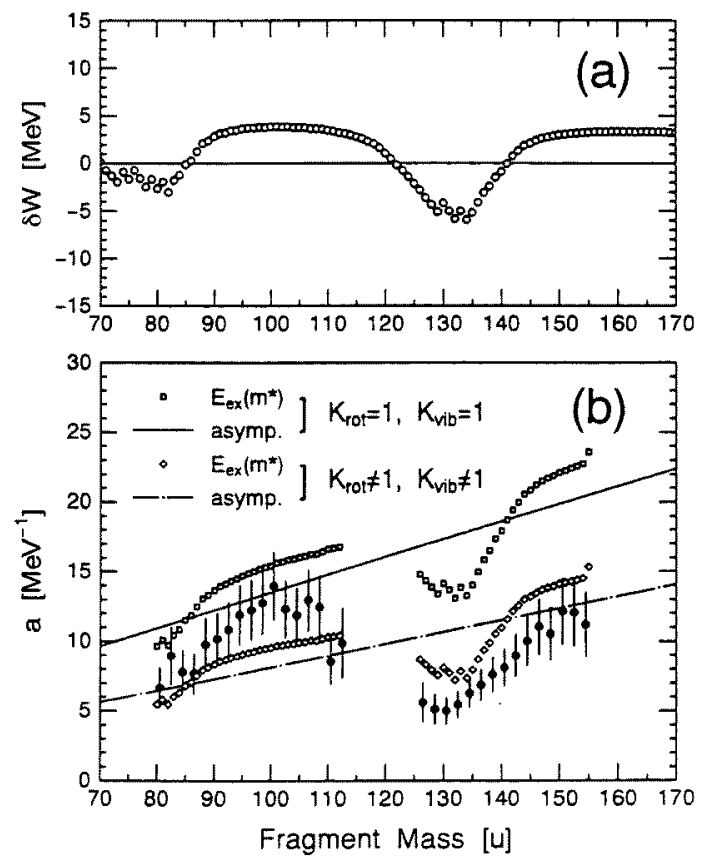

Fig. 6 (a) Shell-correction energies of fission fragments for ${ }^{235} \mathrm{U}\left(n_{t h}, f\right)$ from Myers-Swiatecki ${ }^{(19)}$. (b) Calculated level density parameters using the expression by Iljinov et al. ${ }^{(7)}$

The lozenges and the squares correspond to the results with and without the collective effects, respectively. The dash-dotted $\left(K_{\text {rot }} \neq 1\right.$, $\left.K_{v i b} \neq 1\right)$ and solid lines $\left(K_{\text {rot }}=K_{v i b}=1\right)$ are the asymptotes when the excitation of the fragments increases to infinity.
The calculation demonstrated the dip about $130 \mathrm{u}$ on the LDP curve, and it is seen that the saw-tooth trend of $a\left(m^{*}\right)$ reflects the shape of $\delta W\left(m^{*}\right)$. In the heavy fragment region, the experimental data are close to the calculation with the collective motions. In the light fragment region, the experimental data for $90<m^{*}<105 \mathrm{u}$ lie between the calculations of the two assumptions. Standing on this model, the LDP of this mass region should appear above the asymptotic line, since the fragments in this region have positive shell-correction energies (Eq.(9) and Fig. 6(a)). Therefore, it is reasonable to consider that the light fragments in $90<m^{*}<105 \mathrm{u}$ have collective motions as well as heavy fragments. The experimental data points of very light fragments $\left(m^{*}<90 \mathrm{u}\right)$ and the nearly symmetric light fragments $\left(105<m^{*}<113 \mathrm{u}\right)$ are also rather close to the calculation introducing the collective motion. The decrease of the LDPs due to the collective motions is mainly caused by the rotational motion, since the LD enhancement by this motion $\left(K_{\text {rot }}\right)$ is more predominant than the vibrational enhancement $\left(K_{v i b}\right)$, as described above. It follows that the fission fragments are not spherical but deformed. This is true even for the nuclei near to the double magic number, 132 .

The wash-out of the shell-correction energy at high excitation energy can explain the change of the sawtooth curve of the neutron multiplicity when the fission system is highly excited. Strecker et al. ${ }^{(20)}$ measured the multiplicity of post scission neutrons $\left(\nu_{\text {post }}\right)$, which are thought to come from the fully-accelerated fragment, as a function of the fragment mass for the fission of ${ }^{238} \mathrm{U}$ by proton bombardment. The drastic enhancement of the $\nu_{\text {post }}\left(\mathrm{m}^{*}\right)$ was observed in the heavy mass region of $120<m^{*}<140 \mathrm{u}$, when the bombarding proton-energy is increased to $25.5 \mathrm{MeV}$ from $12.7 \mathrm{MeV}$ (see Fig. 12 in Ref.(20)), whereas the $\nu_{\text {post }}\left(m^{*}\right)$ for light fragments of $90<m^{*}<120 \mathrm{u}$ does not vary so much in this proton energy range. Since the neutron multiplicity is a good index of the fragment excitation energy, the growth of the multiplicity in the heavy region of $120<m^{*}<140 u$ is considered to be the result of the enhancements of $a\left(m^{*}\right)$ and nuclear temperature. It is clear from Eq.(9) and Fig. 6(a) that the increase of $a\left(m^{*}\right)$ for $120<m^{*}<140 \mathrm{u}$ is caused by the wash-out of the strong negative shellcorrection energy. On the contrary, in the light fragment region $\left(90<m^{*}<120 \mathrm{u}\right)$, the change of the neutron multiplicity against the incident proton energy is predicted by the two competing processes, namely (1) the decrement of $a\left(m^{*}\right)$ due to the disappearance of the positive-shell correction energy, and (2) increment of the nuclear temperature caused by the incident particle energy. If the proton energy is increased to $155 \mathrm{MeV}$ for the same reaction of ${ }^{238} \mathrm{U}(p, f)^{(21)}$, the saw-tooth shape of $\nu_{\text {post }}\left(m^{*}\right)$ mostly disappears and the multiplicity increases monotonously against the $m^{*}$. It means that the LDP is converged to the prediction of the Fermi- 
gas model as shown by the line in Fig. 6(b). Such monotonous increase of $\nu_{\text {post }} v s$. mass is also observed in fusion-fission reactions ${ }^{(22)-(24)}$.

\section{Conclusions}

By measuring the multiplicity and emission energy of prompt neutrons from fission fragments following ${ }^{235} \mathrm{U}\left(n_{t h}, f\right)$, the excitation energy and the nuclear temperature of the specified fragment mass were obtained to determine the LDP of the neutron-rich nuclei. The obtained LDP shows the saw-tooth trend against the mass with the minimum value at $130 \mathrm{u}$ which is close to the double magic number of $132 \mathrm{u}$, and is similar to that determined for the ${ }^{252} \mathrm{Cf}(\mathrm{s.f})$ by Budtz-Jørgensen et al. The experimentally obtained $a\left(m^{*}\right)$ and the analysis by using the phenomenological expression of the LDP give the understanding that (1) the shape of the $a\left(\mathrm{~m}^{*}\right)$ can be explained by the shell-correction energy, and (2) the fission fragments have collective motion and hence their LDs are enhanced against the intrinsic density. Since the rotational motion is the dominant factor to the collective enhancement of the $L D$, it can be thought that the fission fragments are deformed.

\section{ACKNOWLEDGMENT}

Fruitful discussion and guidance by Prof. T. Osawa of Kinki University is deeply appreciated. The authors are indebted to Profs. S. Shiroya and K. Kobayashi, Messrs. C. Ichihara, T. Ebisawa and S. Yamamoto of Research Reactor Institute, Kyoto University, and Dr. N. Takahashi of Osaka University for their experimental supports. This work was supported by a Grant-in-Aid for Scientific Research of Ministry of Education, Science, Sports and Culture of Japan. The experiment was performed under the Cooperative Research Program of Research Reactor Institute, Kyoto University.

\section{-References-}

(1) Huizenga, J.R., Moretto, L.G.: Annu. Rev. Nucl. Sci., 22, 472 (1973).

(2) Gilbert, A., Cameron, A.G.W.: Can. J. Phys., 43, 1446 (1965).

(3) Baba, H.: Nucl. Phys., A159, 625 (1970).
(4) Dilg, W., Schantl, W., Vonach, H., Uhl, M.: Nucl. Phys., A217, 269 (1973).

(5) Ignatyuk, A.V., Smirenkin, G.N., Tishin, A.S.: Sov. J Nucl. Phys., 21, 255 (1975).

(6) Beckerman, M.: Nucl. Phys., A278, 333 (1977).

(7) Iljinov, A.S., Mebel, M.V., Bianchi, N., De Sanctis, E., Guaraldo, C., Lucherini, V., Muccifora, V., Polli, E., Reolon, A.R., Rossi, P.: Nucl. Phys., A543, 517 (1992).

(8) Budtz-Jørgensen, C., Knitter, H.-H.: Nucl. Phys., A490, 307 (1988).

(9) van der Ploeg, H., Bacelar, J.C.S., Buda, A., Laurens, C.R., van der Woude, A., Gaardhøje, J.J., Zelazny, Z., van't Hof, G., Kalantar-Nayestanaki, N.: Phys. Rev. $C, \mathbf{5 2}, 1915$ (1995).

(10) Nishio, K., Yamamoto, H., Kimura, I., Nakagome, Y.: Nucl. Instrum. Methods A, 385, 171 (1997).

(11) Nishio, K., Nakagome, Y., Yamamoto, H., Kimura, I.: Submitted to $\mathrm{Nucl}$. Phys. A.

(12) Wagemans, C.: "The Nuclear Fission Process", CRC Press, p.514 (1991).

(13) Apalin, V.F., Gritsyuk, Yu. N., Kutikov, I.E., Lebedev, V.I., Mikaelian, L.A.: Nucl. Phys., 71, 553 (1965).

(14) Maslin, E.E., Rodgers, A.L.: Phys. Rev., 164, 1520 (1967).

(15) Boldeman, J.W., Musgrove, A.R. DE L., Walsh, R.L.: Aust. J. Phys., 24, 821 (1971).

(16) Madland, D.G., Nix, J.R.: Nucl. Sci. Eng., 81, 213 (1982).

(17) Möller, P., Nix, J.R.: At. Data Nucl. Data Tables, 26, 165 (1981).

(18) Bjørnholm, S., Bohr, A., Mottelson, B.: Proc. Symp. on Physics and Chemistry of Fission, IAEA, Vienna, Vol.1, p.367 (1974).

(19) Myers, W.D., Swiatecki, W.J.: Ark. Phys., 36, 343 (1967).

(20) Strecker, M., Wien, R., Plischke, P., Scobel, W.: Phys. Rev. C, 41, 2172 (1990).

(21) Cheifetz, E., Frankel, Z., Galin, J., Lefort, M., Péter, J., Tarrago, X.: Phys. Rev. C, 2, 256 (1970).

(22) Fraenkel, Z., Mayk, I., Unik, J.P., Gorski, A.J., Loveland, W.D.: Phys. Rev. C, 12, 1809 (1975)

(23) Hinde, D.J., Leigh, J.R., Bokhorst, J.J.M., Newton, J.O., Walsh, R.L., Boldeman, J.W.: Nucl. Phys., A472, 318 (1987).

(24) Rossner, H., Hilscher, D., Hinde, D.J., Gebauer, B., Lehmann, M., Wilpert, M., Mordhorst, E.: Phys. Rev. $C, 40,2629$ (1989). 